\title{
Study of cosmic ray ratio of free paths normal and parallel to IMF with muon data
}

\author{
H.S. Ahluwalia ${ }^{1}$ \\ University of New Mexico \\ Albuquerque, NM 87131, USA \\ E-mail:hsa@unm.edu \\ R. Modzelewska \\ Siedlce University, Faculty of Sciences, \\ Konarski str. 208110 Siedlce, Poland \\ E-mail: renatam@uph.edu.pl
}

\begin{abstract}
The galactic cosmic ray (GCR) solar diurnal anisotropy (SDA) data of Nagoya vertical muon telescope $(\mathrm{NagV}-\mathrm{MT})$ are used to compute the ratio $(\boldsymbol{\alpha})$ of GCR free paths normal and parallel to the mean interplanetary magnetic field (IMF) $\mathbf{B}$. They are compared to the corresponding values for the neutron monitors (NMs) of the global network, computed earlier, for three sunspot cycles (21-23) and parts of the other two $(19,24)$. Current modulation theories do not provide any guidance for the value and rigidity dependence of $\boldsymbol{\alpha}$. We use a flat heliospheric current sheet and master equations derived by Ahluwalia and Dorman [1997] for computing $\boldsymbol{\alpha}$ using SDA data for NagV-MT. Preliminary results for its rigidity dependence and correlation with solar activity for positive (p) and negative (n) polarity intervals of $\mathbf{B}$ at 1 AU are noted. Results challenge the validity of old results and conceptual understanding of GCR modulation processes in the heliosphere.
\end{abstract}

36th International Cosmic Ray Conference -ICRC2019-

July 24th - August 1st, 2019

Madison, WI, U.S.A.

\section{${ }^{1}$ Speaker}




\section{Introduction}

Earth acts like a spinning platform immersed in streaming heliospheric galactic cosmic rays (GCRs). Ahluwalia and Dessler [1] suggested that GCR solar diurnal anisotropy (SDA) at earth orbit is related to the dynamics of solar wind (SW) and interplanetary magnetic field (B), leading to formulation of the Parker equation [2] for GCR transport in the heliosphere. SDA has an invariant amplitude $(\sim 0.5 \%)$ over a large rigidity range $(10 \mathrm{GV}<\mathrm{Rm}<300 \mathrm{GV})$ measured at different global sites [3]; Rm is the median value of response of a detector to GCR differential rigidity spectrum.

Using neutron monitor (NM) data from the global network (Rm 20 GV), Ahluwalia et al. [4] give a detailed account of time variations of transport coefficients applicable to GCR interactions with B embedded in turbulent SW at 1 AU. The ratio $(\boldsymbol{\alpha})$ of free paths normal and parallel to mean $\mathbf{B}$ is an important parameter for understanding GCR modulations, including anisotropies. Our computed yearly ratio $(\boldsymbol{\alpha})$ for $\mathrm{NMs} \sim 0.3$ is far higher than values used by others [5-6]. We continue the study with NagV-MT (Rm $=60 \mathrm{GV})$ data. The yearly $\boldsymbol{\alpha}$ is computed empirically using SDA data, a flat heliospheric current sheet (HCS) and the master equations derived by Ahluwalia and Dorman [7].

In a heliospherical polar coordinate system centered on the sun, SDA is a vector (A) with Ar, A $\phi, A \theta$, being radial, east-west, north-south components [8]; Ar, A $\phi$ define the yearly steady state equatorial plane anisotropy. In this paper, we study time variations of $\mathrm{Ar}, \mathrm{A} \phi$ [3] with NagV-MT data for 1971-2017. The rigidity and solar activity dependence of computed $\boldsymbol{\alpha}$ values are compared with those for NMs, where they overlap. The reader is reminded that muon data exhibit pronounced daily and seasonal variations due to changes in atmospheric temperature distribution; daily and monthly hourly rates have to be corrected for the negative temperature effect [ 9 and references therein]. Yearly data are used to eliminate the seasonal effects.

\section{Sunspot numbers (V2)}

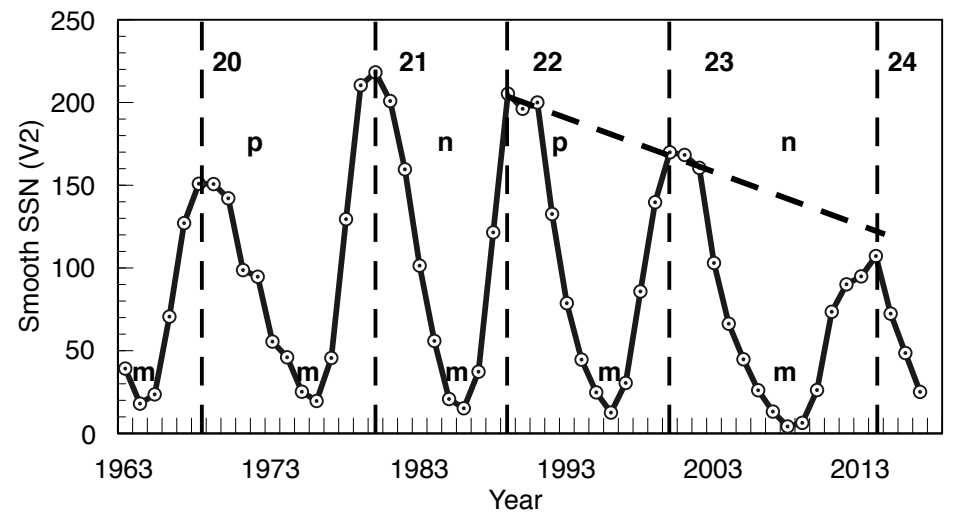

Fig. 1 Yearly smooth SSN(V2) for 1963-2017, vertical dashed lines are drawn through SSN maximum, m indicates minimum; p/n intervals are shown, IMF polarity changes sign near SSN maximum.

Figure 1 depicts yearly smooth sunspot numbers (SSNs) version 2 [10] covering four cycles (20-23) and parts of 19 and 24. The vertical dashed lines are drawn through SSN maximum, $\mathrm{m}$ indicates minimum. The positive (p) and negative (n) IMF polarity intervals at $1 \mathrm{AU}$ are 
covered; B points outward/inward from the sun during $\mathrm{p} / \mathrm{n}$ interval, IMF polarity changes sign near SSN max. For a p-interval protons drift from high latitudes towards HCS, for n-interval they drift from HCS towards higher latitudes [11-12 and references therein].

A descending trend is noted in peak SSNs beginning with cycle 22; it is likely to continue into the future. Livingston and Penn [13] noted a long term weakening of maximum sunspot field since 1992. Penn and Livingston [14] predict that the Cycle 25 will peak with SSN of 7 (a steep decline in solar activity), implying that we may be on the verge of a Maunder-like minimum. This remains to be seen. The physical significance of long-term trends is not clear at this time. The Cycle 24 is the least active cycle recorded, it has not reached minimum value yet; it is also marked by an enhanced north-south asymmetry of the solar polar magnetic field [15].

\section{IMF parameters}

In-situ measurements of $\mathbf{B}$ and solar wind velocity (V) at 1 AU started in October 1963 [16], data are posted at the National Geophysical Data Center website (SPIDR). Figure 2 depicts yearly variations of IMF scalar magnitude (B) and direction $(\psi)$ with respect to Earth-Sun line for 1963-2017. The vertical dashed lines are drawn through SSN maximum, $\mathrm{p} / \mathrm{n}$ intervals are indicated. The following features are noted:

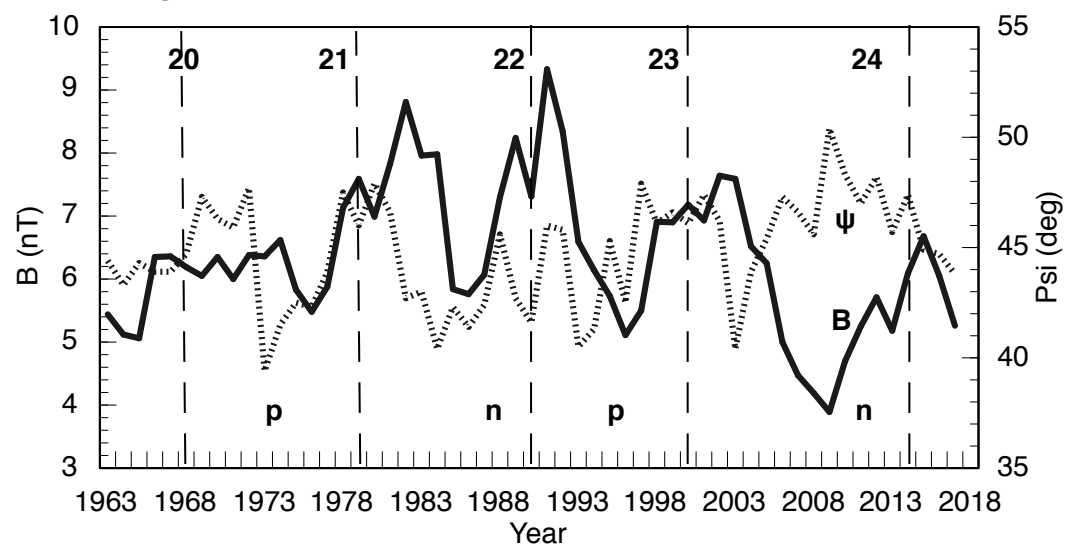

Fig. 2 Yearly B and $\psi$ for 1963-2017, vertical dashed lines are through SSN max; p/n intervals are shown, IMF polarity changes sign near SSN max.

- B value is flat at $\sim 6.5 \mathrm{nT}$ for the Cycle 20 but exhibits $11 \mathrm{y}$ periodicity later; it has two peaks, one near SSN maximum due to magnetic fields carried by ICMEs and a post maximum peak due to high-speed SW streams.

- After the Cycle 20, minimum B has smaller values for each cycle, reaching the smallest value recorded in 2008 at the Cycle 23 minimum (of long duration). This corresponds to a similar trend in SSN minima (m) in Fig. 1; expected since SSNs and B are correlated [17].

- Yearly value of $\psi$ varies within a narrow range $\left(40^{\circ}-50^{\circ}\right)$; IMF coverage is very poor for early years. In this paper, we use data for $\mathrm{p} / \mathrm{n}$ intervals to compute $\boldsymbol{\alpha}$ from corresponding SDA components, hence a need for adequate IMF coverage.

\section{Analysis procedure}

The pressure corrected hourly rate for a detector (i) is used to compute deviations expressed as $\%$ of daily mean rate in local time (LT). The harmonic analysis [18] of the deviations gives 
the amplitude of the diurnal variation $\left(\mathrm{a}_{\mathrm{i}}, \%\right)$ and its phase (LT, $\left.\mathrm{h}\right)$. The data are corrected for the geomagnetic bending (GB) and the orbital effect [19-20]. Yearly $a_{i}$ is converted to the equatorial plane anisotropy amplitude (A, \%) for a limiting GCR rigidity $(\mathrm{Rc})$, following the procedure developed by Ahluwalia and Riker [21], as follows:

$$
\mathrm{a}_{\mathrm{i}}=\int_{\mathrm{Ro}}^{\mathrm{Rc}} \frac{\delta \mathrm{D}(\mathrm{R})}{\mathrm{D}(\mathrm{R})} \mathrm{W}_{\mathrm{i}}(\mathrm{R}) \mathrm{dR}
$$

$\mathrm{Wi}(\mathrm{R})$ is the coupling function [22] for detector i, Ro is its effective vertical threshold rigidity, $\delta \mathrm{D}(\mathrm{R}) / \mathrm{D}(\mathrm{R})$ is the variational spectrum defined by Ahluwalia and Riker [21] as:

$$
\delta \mathrm{D}(\mathrm{R}) / \mathrm{D}(\mathrm{R})=\mathrm{AR}^{\gamma} \operatorname{Cos} \lambda \mathrm{a} \text {, if } \mathrm{R} \leq \mathrm{Rc}
$$

$\lambda \mathrm{a}$ is the asymptotic latitude of viewing for detector $\mathrm{i}$, and $\gamma=0$ [19]; A is insignificant above Rc and at poles $\left(\lambda \mathrm{a}=90^{\circ}\right)$. The value of $\mathrm{Rc}$ is related to yearly $\mathrm{B}$, it changes from one year to next [23]. Munakata et al. [6] use Rc $=100 \mathrm{GV}$ for NagV-MT data (35N 137E). We use Rc value computed from the regression relation between Rc, B [24] for the entire period (1971-2017) for NagV-MT $($ Ro $=10 \mathrm{GV})$ to compare our results with theirs; the coupling function (W) derived by Murakami et al. [25-26] for NagV-MT are used to compute A and resolved into the east-west $(\mathrm{A} \phi)$ and radial (Ar) components [3]; data are corrected for negative temperature effect using empirical vector in $6 \mathrm{~h}$ LT direction with an amplitude of $0.11 \%$ [9].

\section{Nagoya V-MT data}

Yearly diurnal amplitude (\%) and phase (LT, h) are plotted in Figure 3 and yearly SDA amplitude (\%) and direction in free space are plotted in Figure 4 for 1971-2017. Times of maxima exhibit a strong Hale (22y) cycle, reported by Thambyahpillai and Elliott [27];

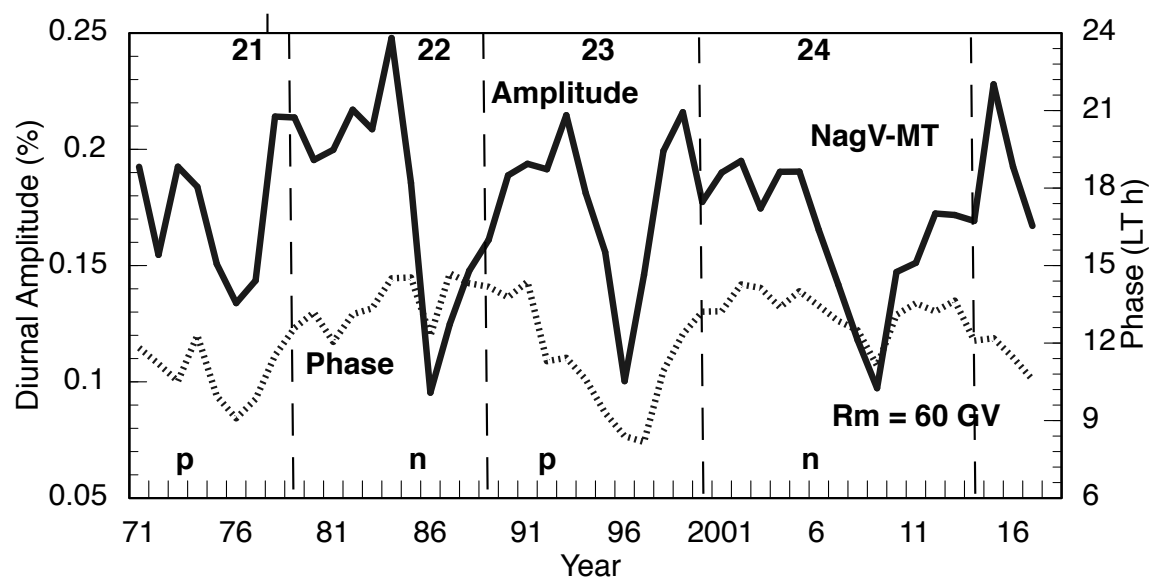

Fig. 3 Yearly NagV-MT diurnal amplitude (\%) and phase (LT h) for 1971-2017, vertical dashed lines are through SSN max; IMF polarity changes sign near SSN max. 


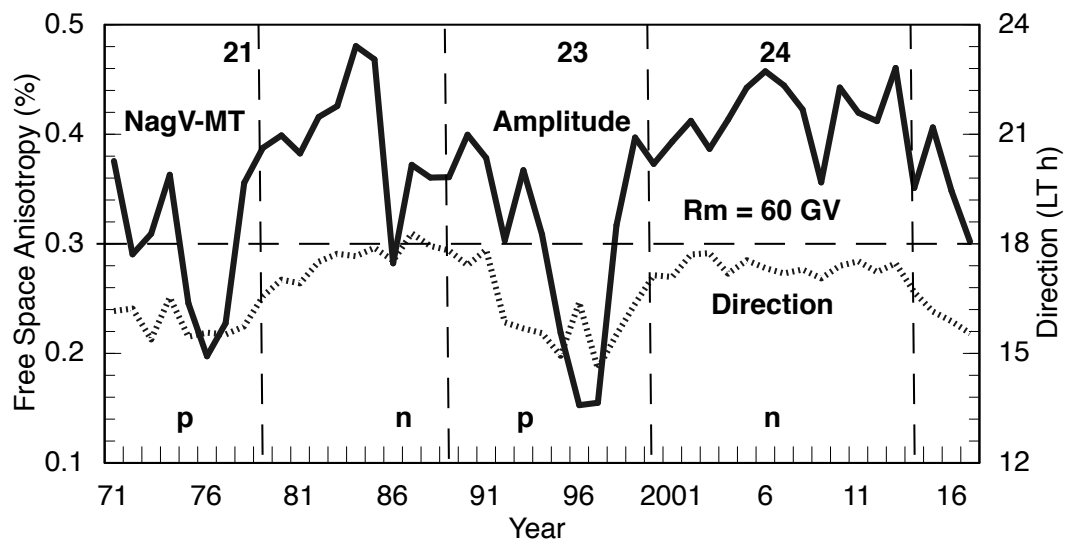

Fig. 4 Yearly equatorial plane anisotropy (amplitude and direction) for NagV-MT for 1971-2017.

amplitudes in both have low values near SSN min in 1976, 1986, 1996, and 2008, in good agreement with Munakata et al. [6] trends. Ahluwalia [3] showed that A has an invariant value $0.5 \%$ for NMs and MTs $(20 \mathrm{GV}<\mathrm{Rm}<300 \mathrm{GV})$ for $1965-1970$.

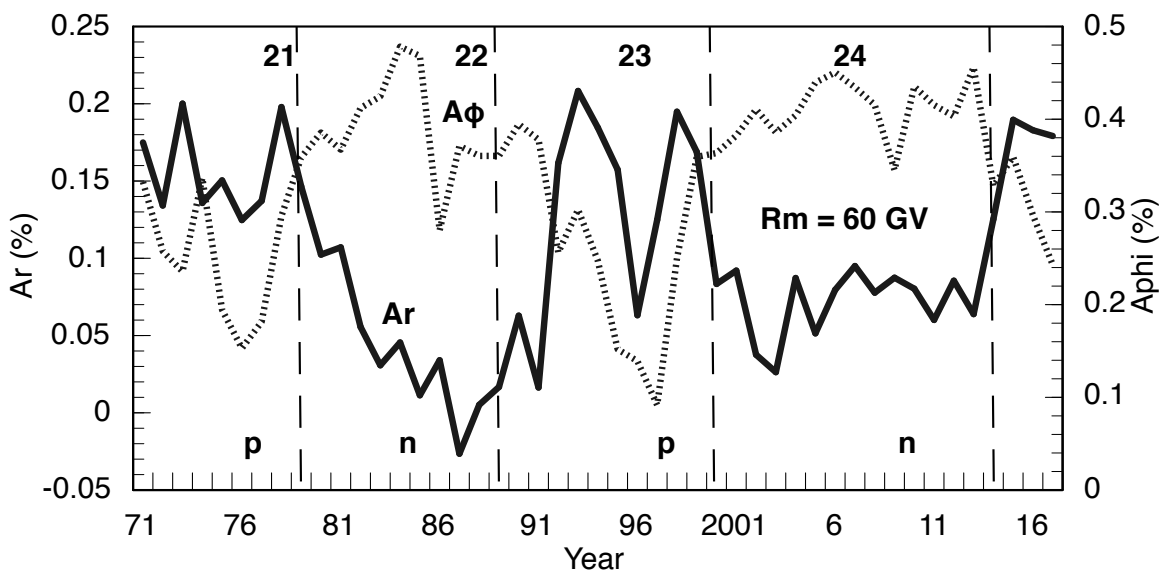

Fig. 5 Yearly radial (Ar) and east-west (A $\phi$ ) anisotropies for 1971-2017, vertical dashed lines are through SSN max; IMF polarity changes sign near SSN max.

Figure 5 depicts yearly radial (Ar) and east-west (A $\varphi$ ) anisotropies for 1971-2017; vertical dashed lines are through SSN max, IMF polarity changes sign near SSN max.

We note that:

- $\mathrm{Ar}<\mathrm{A} \varphi$ and has higher values in $\mathrm{p}$ - than in $\mathrm{n}$ - intervals, exhibiting Hale cycle (22y) with lower values near SSN min. Since $\mathrm{Ar} \neq 0$, the spherical symmetry approximation of Parker equation is invalid for SDA study.

- A $\varphi$ is higher $(\sim 0.45 \%)$ near SSN maxima at 1979, 1989, 2000, 2014, and lower near SSN minima at 1976, 1986, 1996, 2008. It has lower values in p- than in n-intervals (Hale cycle). The time variations of $\mathrm{Ar}, \mathrm{A} \varphi$ are similar to those for NMs but amplitudes are larger.

Ahluwalia and Dorman [7] showed that for a flat HCS:

$$
\begin{array}{lll}
\left(\mathrm{Ar}^{\mathrm{b}}+\mathrm{Ar}^{\mathrm{n}}\right) / 2=3 / \mathrm{v}(\mathrm{CV}-\mathrm{KrrGr}) & (3), & \mathrm{Krr}=\mathrm{K} \| \operatorname{Cos}^{2} \psi+\mathrm{K} \perp \operatorname{Sin}^{2} \psi \\
\left(\mathrm{A} \varphi^{\mathrm{b}}+\mathrm{A} \varphi^{\mathrm{n}}\right)=(1-\alpha) \lambda \| \mathrm{GrSin} 2 \psi & (4), & \lambda \| \mathrm{Gr}=-\mathrm{Ar}+3 \mathrm{CV} / \mathrm{v}+\mathrm{A} \varphi \operatorname{Tan} \psi
\end{array}
$$

superscripts $\mathrm{p} / \mathrm{n}$ apply to positive/negative IMF polarities; Gr is radial density gradient directed 
away from the sun; Krr is radial diffusion coefficient; $\lambda \|$ is free path parallel to mean $\mathbf{B}$; V is solar wind velocity; $\mathrm{v}(\sim \mathrm{c})$ is GCR velocity; $\psi$ is IMF spiral angle; $\mathrm{C} \approx 1.5$ is the ComptonGetting coefficient. Eqns 3, 4 include diffusion-convection terms only.

Figure 6 depicts yearly variations of modulation parameter $\lambda \| \mathrm{Gr}(\%)$ for 1971-2017 for $\mathrm{NagV}$ MT. It exhibits weak 11y and strong 22y dependence, with lower values near SSN min (m). It has the same form as for NMs for an overlapping period, indicating that the product $\lambda \| \mathrm{Gr}$ is independent of GCR rigidity; confirming and extending Ahluwalia [12, Fig. 3] inference for 1965-1990.

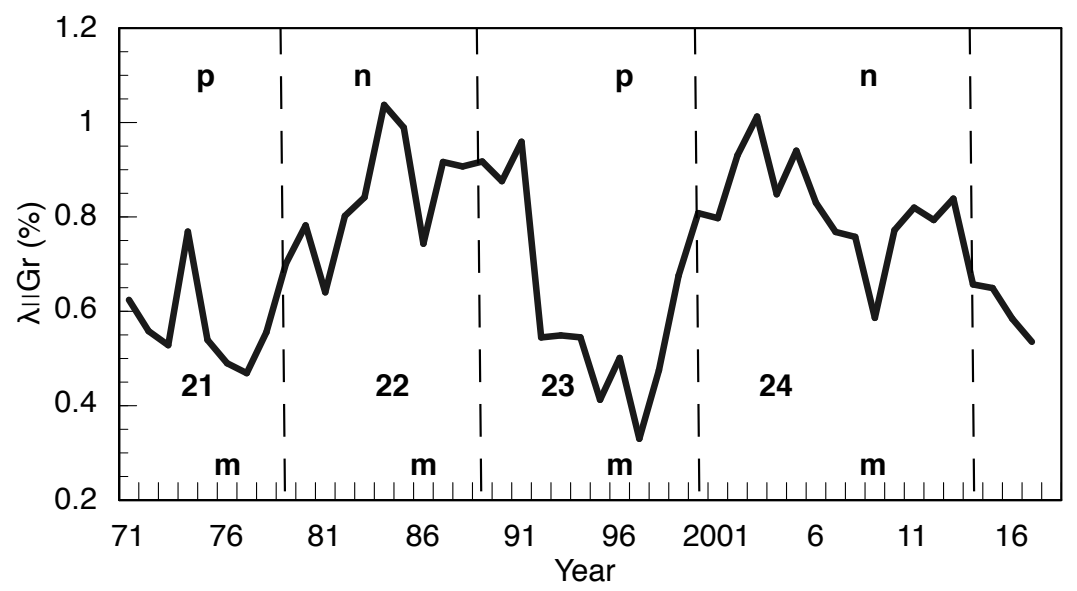

Fig. 6 Yearly variations of $\lambda \| \mathrm{Gr}(\%)$ for 1971-2017 for NagV-MT, vertical dashed lines are through SSN max; IMF polarity changes sign near SSN max.

Yearly $\boldsymbol{\alpha}$ values are obtained by subdividing Ar, A $\varphi$ into p/n intervals and using eqns (3), (4) which include diffusion-convection terms only. The computed $\boldsymbol{\alpha}$ values are displayed in Fig. 7 along with those available for NMs at different Rm values; $\boldsymbol{\alpha}$ is high for $\mathrm{p}$ - and low for $\mathrm{n}$ intervals, compared to being nearly flat for NMs. The $\boldsymbol{\alpha}$ values for NM2 $(\mathrm{Rm}=25 \mathrm{GV})$ are larger than those for NM1 $(\mathrm{Rm}=15 \mathrm{GV})$, implying a rigidity dependence. The $\boldsymbol{\alpha}$ values for NagV-MT are low near SSN max (vertical dashed lines) as is the case for NMs and high near SSN min (m), when Rc values are known to be lower than $100 \mathrm{GV}$ [27]. Munakata et al. [6] computed modulation parameters assuming $\boldsymbol{\alpha}=0.01$ following Bieber and Chen [5], without additional justification. Therefore, some of their inferences about the derived modulation parameters and their time variations are questionable. Chen and Bieber [29] noted that GCR gradients and $\lambda \|$ depend strongly upon $\boldsymbol{\alpha}$. They reviewed several alternate methods reported in the literature for treating $\boldsymbol{\alpha}$. An empirical test devised by them to obtain consistent physical results led them to conclude that there is a limiting value of $\boldsymbol{\alpha}=0.16$; for higher values they were unable to make observed SDA consistent with GCR streaming equations. Following a similar train of thoughts Hall et al. [30] obtained an upper limit for $\boldsymbol{\alpha} \sim 0.3$ for NMs (Rm $=17$ $\mathrm{GV}$ ) for n-interval and an upper limit for $\boldsymbol{\alpha} \sim 0.8$ for $\mathrm{p}$-interval for Hobart underground MTs (Rm $=185 \mathrm{GV}$ ) for 1957-1990. We confirm their findings for two additional Schwabe cycles. 


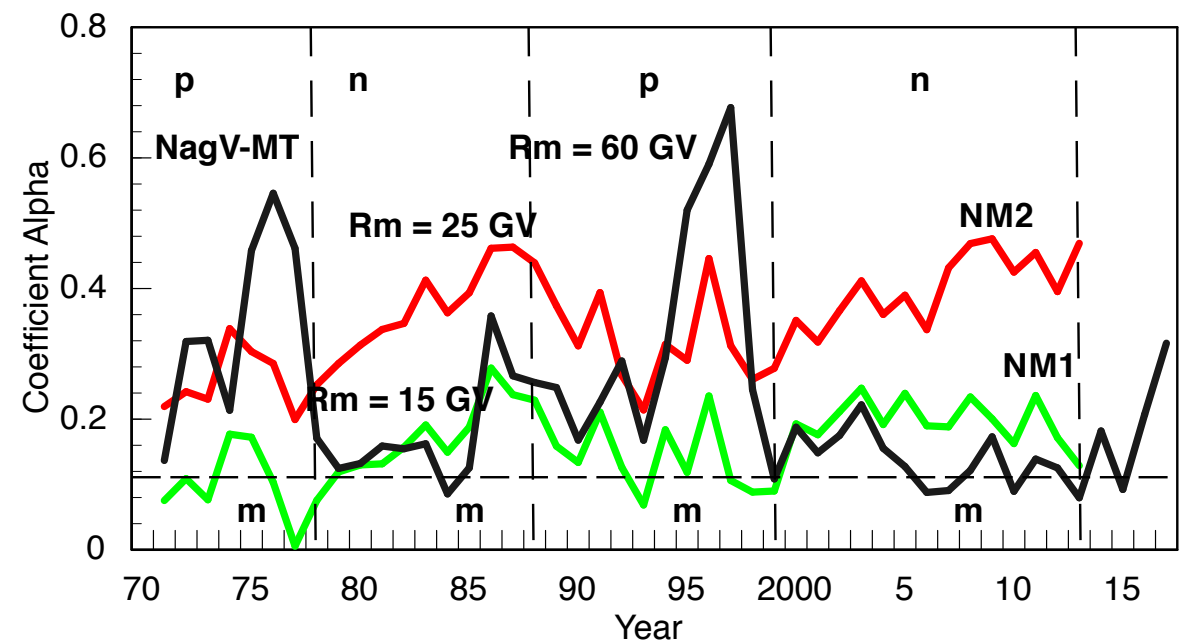

Fig. 7 Yearly $\boldsymbol{\alpha}$ values for NagV- MT $(\mathrm{Rm}=60 \mathrm{GV})$ for 1971-2017 along with those for NMs reported earlier [4].

\section{Acknowledgements}

We thank Michael Fikani and Roger Ygbuhay for technical assistance and the providers of solar \& interplanetary data. HSA thanks Kazuoki Munakata for NagV-MT data as well as for discussions; he is also grateful to the U.S. Air Force Office of Scientific Research for Summer Faculty Research Fellowship at the Space Vehicles Directorate, Kirtland Air Force Base, NM.

\section{References}

[1] H. S. Ahluwalia and A.J. Dessler, Planet. Space Sci. 9 (1962) 195-210

[2] E. N. Parker, Planet. Space Sci. 13 (1965) 9-49

[3] H.S. Ahluwalia, Planet. Space Sci. 36 (1988) 1451-1459

[4] H. S. Ahluwalia et al., J. Geophys. Res. Space Phys. 120 (2015) 8229- 8246

[5] J.W. Bieber and J. Chen, Astrophys. J. 372 (1991) 301-313

[6] K. Munakata, M. Kozai, C. Kato and J. Kota, Astrophys. J. 791 (2014) 22(16pp)

[7] H. S. Ahluwalia and L. I. Dorman, J. Geophys. Res. 102 (1997) 17 433-17 443

[8] J.F. Riker and H.S. Ahluwalia, Planet. Space Sci. 35 (1987) 1117-1122

[9] H.S. Ahluwalia, J. Geophys. Res. 98 (1993) 11513-11519

[10] F. Clette et al. Space Sci. Rev. 186 (2014) 35-103

[11] J. Kota and J.R. Jokipii, Astrophys. J. 265 (1983) 573-581

[12] H.S. Ahluwalia, J. Geophys. Res. 99 (1994) 23515-23521

[13] W. Livingston and M. Penn, EOS 90 (2009) 30

[14] M. Penn and W. Livingston, arXiv:1009.0784v1 2010 [astro-ph.SR]

[15] H. S. Ahluwalia, J. Atmos and Solar-Terr. Phys. 176 (2018) 57-60

[16] C.W. Snyder, M. Neugebauer and U.R. Rao, J. Geophys. Res. 68 (1963) 6361-6370

[17] H. S. Ahluwalia, Adv. Space Res. 52 (2013) 2112-2118

[18] D. Gubbins, Time Series Analysis and Inverse Theory for Geophysicists, Cambridge University Press (2004) Cambridge, U. K.

[19] H. S. Ahluwalia and J. H. Ericksen, Acta Phys. Acad. Sci. Hung. 29 (Suppl. 2) (1970) 139-146 
[20] H. S. Ahluwalia and J. H. Ericksen, J. Geophys. Res. 76 (1971) 6613-6627

[21] H. S. Ahluwalia and J. F. Riker, Planet. Space Sci. 35 (1987) 45-50

[22] Dorman, L. I., Cosmic Ray Variations, State Publishing House for Tech. and Theoretical Literature (1957) Moscow

[23] H. S. Ahluwalia, Geophys. Res. Lett. 19 (1992) 633-636

[24] H. S. Ahluwalia, Geophys. Res. Lett. 27 (2000) 617-620

[25] K. Murakami, K. Nagashima, S. Sagisaka, Y. Mishima and I. Inoue, Il Nuovo Cimento 2C N.5 (1979) 635-651

[26] K. Murakami, S. Sagisaka, Y. Mishima, I. Inoue and K. Nagashima, Rept. Cosmic Ray Res. Lab. 6 (1981) Nagoya Univ.

[27] T. Thambyahpillai and H. Elliott, Nature 171 (1953) 918-920

[28] R.C. Ygbuhay, Galactic cosmic ray transport in the heliosphere, PhD dissertation, University of New Mexico (2015)

[29] J. Chen and J. W. Bieber, Astrophys. J. 405 (1993) 375-389

[30] D.L. Hall, M.L. Duldig and J.E. Humble, Proc. $24^{\text {th }}$ Int. Cosmic Ray Conf. 4 (1995) 607-610 